\title{
Quand deux chromosomes homologues viennent d'un seul parent... les disomies uniparentales en pathologie
}

La nécessité des deux génomes parentaux pour le développement de l'embryon est bien établie chez la souris comme chez l'homme. Un exemple caricatural est fourni par la môle hydatiforme, souvent diploïde et caryotypiquement normale, dont tous les chromosomes sont d'origine paternelle; cette "androgenèse » entraîne une hypertrophie du trophoblaste avec absence souvent complète de tissus foetaux. Quant à la parthénogenèse, on n'a pas pu la rendre viable chez la souris et elle n'a pas été observée dans l'espèce humaine. Mais si, au lieu de l'ensemble du génome, une seule paire de chromosomes homologueś provient d'un seul parent, des phénomènes pathologiques se produiront-ils ? La disparition d'un des chromosomes homologues, accompagnée de son remplacement par son partenaire, et aboutissant à une homozygotie pour un des chromosomes, est un phénomène bien connu en cancérologie. Les exemples les mieux étudiés sont ceux du rétinoblastome et de la tumeur de Wilms; au cours de cette dernière peut survenir une perte préférentielle d'allèles maternels [1]. En 1988, pour la première fois, des chercheurs de Houston (TX, USA) et Toronto (Canada) [2] ont décrit une disomie uniparentale en l'absence de cancer (voir $m / s, n^{\circ} 2$, vol. 4, p. 127). Il s'agissait d'une femme atteinte de mucoviscidose, à laquelle le chromosome 7 paternel n'avait pas été transmis, et dont les deux chromosomes 7 , d'origine maternelle, étaient identiques. A la différence du cancer, ce sont ici toutes les cellules de l'organisme qui possèdent cette homozygotie. Tout récemment, un deuxième cas a été décrit en Israèl chez un garçon de quatre ans [3]. Bien entendu, dans les deux cas, l'hypothèse de non-paternité du père légal avait été éliminée. Avec ce deuxième $\mathrm{m} / \mathrm{s} n^{\circ} 1$ vol. 6, janvier 90 exemple, ce qui apparaissait comme une curiosité exceptionnelle va-t-il acquérir droit de cité, et devenir un mécanisme dont l'éventualité devra être prise en compte?

Les concepts de disomie uniparentale (DUP) (les deux chromosomes homologues provenant d'un seul parent) et d'isodisomie (les deux chromosomes étant identiques, provenant du même chromosome qui aurait subi une duplication) ont été définis théoriquement il y a dix ans par Engel [4]. Des disomies ont été réalisées expérimentalement chez la souris, montrant que la présence de deux autosomes d'un même parent provoquait ou non des troubles pathologiques selon le chromosome en cause (empreintes parentales ou génomiques), décrits récemment en détail par Babinet $e t$ al. dans $m / s$ [5]. L'inégalité des rôles du père et de la mère est également suggérée par les différences de gravité, ou de date d'apparition, selon le parent dont certaines maladies sont héritées $(\mathrm{m} / \mathrm{s}$, $n^{\circ} 2$, vol. 2, p.103 et Tableau I).
Il est donc permis de se demander si d'éventuelles disomies ne pourraient à elles seules être génératrices d'états pathologiques.

Il est possible de calculer approximativement la fréquence de la DUP en supposant que la cause la plus habituelle devrait être la «complémentation génétique », c'est-à-dire l'union d'un gamète disomique avec un nullisomique. On évalue à environ $1 \%$ chacun les pourcentages de nullisomiques et de disomiques dans les spermatozoïdes et à environ $12 \%$ dans les ovocytes. En supposant que la probabilité est la même pour tous les autosomes, Warburton [6] aboutit à la conclusion qu'une conception sur 30000 pourrait comporter une isodisomie. Bien des inconnues obligent naturellement à ne donner ce chiffre qu'à titre indicatif.

C'est au cours d'études familiales entreprises à partir de malades atteints de mucoviscidoses reconnues que des anomalies furent constatées : employant des sondes du chromosome 7 voisines du locus $\mathrm{CF}$, on

Tableau 1

EFFET DE L'ORIGINE PARENTALE SUR CERTAINES MALADIES

\begin{tabular}{|l|c|l|}
\hline Maladie & Chromosome & Observations \\
\hline Chorée de Huntington & 4 & $\begin{array}{l}\text { Début précoce associé à transmis- } \\
\text { sion paternelle } \\
\text { Début précoce si transmission } \\
\text { paternelle } \\
\text { Forme congénitale à transmission } \\
\text { maternelle }\end{array}$ \\
Myotonie de Steinert & 19 & $\begin{array}{l}\text { Plus sévère en cas de transmission } \\
\text { maternelle } \\
\text { Début précoce si transmission } \\
\text { maternelle } \\
\text { Disomie paternelle }\end{array}$ \\
Neurofibromatose I & 17 ibromatose II & Tous \\
Môle hydatiforme & &
\end{tabular}

Modifié d'après [5]. 
constatait qu'avec des polymorphismes de restriction pour lesquels le père était homozygote pour un allèle, l'enfant l'était pour un autre; le cas de Spence et al. [2] présentait la difficulté supplémentaire que, la mère étant morte, son $\mathrm{ADN}$ ne pouvait servir de référence, et il fallut des recherches minutieuses pour éliminer l'hypothèse d'une relation incestueuse avec un de ses frères, susceptible d'expliquer une homozygosité apparente. Un examen de nombreux loci du chromosome 7 permit de se persuader que, comme dans le cas de Voss et al. [3], l'homozygosité était complète. C'est ainsi que ces malades, dont un seul des parents était hétérozygote pour la mucoviscidose, et qui n'avaient en principe aucune chance d'en être frappés, ont eu la malchance de voir sélectionné, en double dose, le seul chromosome porteur de l'anomalie.

Les mécanismes théoriquement responsables d'une DUP sont nombreux. Elle peut résulter d'une erreur précédant ou suivant la fertilisation. Une complémentation gamétique implique, nous l'avons dit, fertilisation entre gamètes nullisomiques et disomiques donnant un zygote uniparental, mais en principe non isodisomique. Il en va de même d'une trisomie ayant perdu le chromosome unique venant du deuxième parent. Une isodisomie, au contraire, peut résulter d'un zygote monosomique « rescapé » grâce à une duplication secondaire, mais aussi d'une erreur survenant après la fertilisation par non-disjonction suivie de duplication (figure 1). On conçoit qu'il ne soit pas toujours aisé de remonter à la source de telles anomalies.

Le champ possible des disomies uni- parentales n'a probablement pas encore été complètement exploré.

(1). Mucoviscidose. Warburton [6], se fondant sur la fréquence probable des isodisomies et celle du gène $C F$, calcule que l'on pourrait s'attendre à un pourcentage d'isodisomie 7 dans la mucoviscidose de l'ordre de 1 sur 10000 . Or Voss et al. [3] font remarquer que déjà deux cas d'isodisomie ont été reconnus, sur le nombre total de familles explorées, qu'ils évaluent à 2000 . Le recensement va certes continuer et sa vitesse s'accélérer, mais il est peut-être un moyen d'obtenir une réponse plus rapide à la question de la fréquence des isodisomies dans cette maladie: les deux sujets disomiques ont un caractère commun, une taille très inférieure à la normale; en focalisant une recherche sur les mucoviscidosiques de petite taille, il serait possible de

\begin{tabular}{|c|c|c|c|c|c|}
\hline & Normal & $\begin{array}{l}\text { Complémentation } \\
\text { gamétique }\end{array}$ & $\begin{array}{l}\text { Trisomie } \\
\text { a disomie }\end{array}$ & $\begin{array}{c}\text { Monosomie } \\
\text { à disomie }\end{array}$ & $\begin{array}{l}\text { Post-fertilisation } \\
\text { non-disjonction }\end{array}$ \\
\hline \multicolumn{6}{|c|}{ Gamètes } \\
\hline \multicolumn{6}{|l|}{ Zygote } \\
\hline \multicolumn{6}{|l|}{ Mitose } \\
\hline \multicolumn{6}{|l|}{ Soma } \\
\hline & & $\begin{array}{r}\text { DUP } \\
\text { HD }\end{array}$ & $\begin{array}{l}\text { JUP } \\
\text { HD }\end{array}$ & $\begin{array}{r}\text { DUP } \\
\text { ID }\end{array}$ & $\begin{array}{l}\text { DUP } \\
\text { ID }\end{array}$ \\
\hline
\end{tabular}

Figure 1. Mécanismes capables d'entraîner une disomie uniparentale (DUP) et une isodisomie (ID). HD = hétérodisomie. (Modifié d'après [2].) 
vérifier l'existence ou l'absence de cas supplémentaires. Si la réponse était positive, deux autres questions pourraient être posées: la petite taille n'étant probablement pas due à la mucoviscidose elle-même, trouveraiton chez les sujets inhabituellement petits, sans cause génétique ni métabolique décelable, des cas d'isodisomie, ou du moins de chromosome 7 uniparental ? La deuxième question provient du fait que dans les deux cas le chromosome 7 est d'origine maternelle; en est-il toujours ainsi, ce qui suggèrerait qu'un 7 uniparental d'origine paternelle ne serait pas viable?

(2). Autres chromosomes ; isodisomies partielles, mosaïques, chromosomes sexuels. On commence à trouver dans la littérature d'autres cas de DUP totale ou partielle d'un chromosome en dehors du cancer.

(a). Le syndrome de Prader-Willi associe acromicrie, hypotonie, obésité et hypogonadisme. Dans $60 \%$ des cas, il comporte une délétion en 15q11q13, presque toujours d'origine paternelle, alors que la même délétion, transmise par la mère, est à l'origine du syndrome d'Angelman, manifesté par un retard mental, des convulsions, une démarche ataxique. Nicholls et al. [7] ont entrepris l'étude de l'ADN de malades sans délétions décelables. Dans deux familles de syndrome de Prader-Willi - et probablement, d'après des essais préliminaires, dans quatre autres le chromosome 15 est d'origine uniquement maternelle; il s'agit d'une hétérodisomie puisque les deux chromosomes de la mère sont présents, mais il n'y a aucune contribution paternelle, au moins pour la portion ql l-q13, la limite de la disomie n'étant pas encore complètement déterminée. Il semble donc que l'absence d'allèles paternels dans la région qll-ql3 soit pathogène, et que dans ce cas on puisse considérer une «empreinte génomique » comme confirmée. La question de savoir si l'absence, dans cette même région, d'allèles maternels suffit à provoquer le syndrome d'Angelman peut être également posée.

(b). Mongolisme et mosaïcismes chromosomiques. La possibilité de DUP au cours d'une anomalie chro$\mathrm{m} / \mathrm{s} n^{\circ} 1$ vol. 6, janvier 90 mosomique est illustrée par un travail japonais datant de 1984 et utilisant uniquement des méthodes cytogénétiques [8]. Chez trois sujets, on conclut qu'une erreur au cours de la méiose avait donné naissance à la trisomie 21 , suivie lors d'une mitose par la perte d'un chromosome 21 dans certaines cellules. Chez deux, et peut-être chez les trois malades, les chromosomes 21 des cellules diploïdes provenaient d'un seul parent. Un tel passage de trisomie à disomie pourrait expliquer les cas où une trisomie avec ou sans mosaïque a pu être constatée sur une biopsie du trophoblaste et pas sur un examen plus tardif ; c'est ainsi qu'une trisomie 13 avec mosaïque et une trisomie 16 sans mosaïque ont été suivies de ponctions amniotiques normales [9].

(c). Chromosomes sexuels. Une disomie uniparentale (terme peut-être incorrect pour des chromosomes non autosomes, mais il n'existe pas d'expression spécifique) pourrait affecter les chromosomes sexuels, notamment par nullisomie d'un des gamètes, l'autre introduisant en bloc les deux X maternels chez une fille, ou l'ensemble XY du père chez un garçon. Un exemple frappant vient d'en être relaté par une équipe française [10]. Un garçon hémophile avait un père et un oncle paternel également hémophiles. L'analyse de 15 marqueurs d'ADN a prouvé que ce garçon avait hérité de l'X de son père, et non de sa mère, qui d'ailleurs ne semble pas transmettrice, alors que pour plusieurs autosomes on a pu éliminer toute DUP. Il s'agit donc d'une disomie uniparentale (mais non évidemment d'une isodisomie), impliquant la fertilisation d'un ovocyte nullisomique pour l'X par un spermatozoïde disomique pour les chromosomes sexuels. En dehors de son hémophilie, ce garçon paraît normal ; il n'y a donc pas de phénomène d'empreinte parentale lorsqu'un garçon ne reçoit pas d'X de sa mère.

(3). Translocations. Dans les translocations robertsoniennes deux chromosomes fusionnent par leurs extrémités. On connaît des cas de translocations de chromosomes homologues, notamment entre les deux chromosomes 22. Cette translocation est équilibrée et ne crée pas de perturbations cliniques. Mais à la génération suivante les deux chromosomes 22 fusionnés sont transmis ensemble; comme l'autre parent fournit un chromosome 22, l'enfant aura une trisomie 22. Or on connaît deux cas de translocation $22 ; 22$ qui ont donné naissance à des filles normales par disomie uniparentale, l'autre parent ne contribuant pas, ou son chromosome 22 ayant été perdu au cours d'une mitose initiale (dans [2]).

Une observation encore plus surprenante a été relatée en 1982 [11]. Un examen prénatal a révélé l'existence, chez un fœetus apparemment normal à l'échographie, d'une translocation robertsonienne 14;21 homozygote. Or si le père était bien porteur de cette translocation à l'état hétérozygote, la mère ne l'était pas; on peut donc faire l'hypothèse qu'une double dose du 14;21 paternel a été transmise à l'enfant, ce qui impliquerait, puisqu'il n'y avait pas de déséquilibre, qu'il n'y avait aucune contribution maternelle pour deux chromosomes, 14 et 21 . Cette hypothèse, qui n'est naturellement pas la seule, a été soulevée mais n'a pu être vérifiée à cette date. La grossesse a été interrompue à 18 semaines, et l'autopsie n'a montré aucune anomalie décelable à l'examen macro- et microscopique.

Conclusion. Dans cet exposé, nous nous sommes limités aux exemples qui ne comportent pas de déséquilibres ni de délétions décelables, afin de cerner uniquement les effets, s'il en existe, de l'origine paternelle ou maternelle des événements constatés. Puisqu'une expérimentation très poussée a été effectuée chez la souris, on s'est efforcé[5] de retrouver la correspondance, sur un de ses chromosomes, des loci pathologiques humains. Ces travaux donneront sans doute des renseignements importants mais sont encore très fragmentaires. En attendant, est-il possible de conclure dès maintenant à l'existence chez l'homme de chromosomes qui doivent provenir des deux parents pour prévenir des conséquences pathologiques ? Deux candidats peuvent déjà postuler : le chromosome 7, pour lequel l'absence 
d'allèles paternels semble à l'origine d'un défaut de croissance; le chromosome 15, dont la région q11-q13 doit provenir des deux parents pour éviter des troubles graves. Deux autres semblent à l'abri du phénomène d'empreinte parentale, le chromosome 22 et le chromosome $\mathrm{X}$.

A l'avenir les chercheurs devront être à l'affût des malades homozygotes dont un des parents ne semble pas être transmetteur, ainsi que des trisomies qui donnent secondairement des disomies; peut-être faudrait-il aussi reconsidérer certaines recombinaisons génétiques difficilement explicables. Enfin, l'on peut imaginer qu'une disomie uniparentale pour une région chromosomique soumise à une forte «empreinte parentale » aboutisse, comme chez la souris [5], à des troubles létaux du développement embryonnaire.

Peut-être cette hypothèse pourraitelle être vérifiée en analysant des produits d'avortements spontanés sans anomalie cytogénétique. Les chercheurs pourraient alors être gui- dés par la connaissance des homologies entre les régions soumises chez la souris à une telle empreinte et leurs équivalents humains.

Nous ne sommes qu'à l'aube des travaux sur les disomies uniparentales, et nul ne peut prévoir l'extension que ce mécanisme pourra prendre dans l'avenir.

\section{Jean-Claude Dreyfus}

\section{RÉFÉRENCES}

1. Junien C, Henry I. Bras court du chromosome 11: empreinte parentale différentielle, tumorigenèse et perte d'allèles. médecine/ sciences $1989 ; 5: 480-8$

2. Spence JE, Perciaccante RC, Grig GM, et al. Uniparental disomy as a mechanism for human genetic disease. Am J Hum Genet $1988 ; 42: 217-26$

3. Voss R, Ben-Simon E, Avital A, et al. Isodisomy of chromosome 7 in a patient with cystic fibrosis : could uniparental disomy be common in humans? Am J Hum Genet $1989 ; 45: 373-80$.

4. Engel E. A new genetic concept: uniparental disomy and its potential effect, isodisomy. Am J Med Genet 1980; 6 : 137-43.

5. Babinet C, Barra J, Renard JP. Le marquage et l'expression différentiels des génomes paternel et maternel : deux conditions nécessaires pour le développement à terme de l'embryon de souris. médecinel sciences $1989 ; 5: 8-15$.

6. Warburton D. Editorial: uniparental disomy: a rare consequence of the high rate of aneuploidy in human gametes. Am J Hum Genet 1988; 42 : 215-6.

7. Nicholls RD, Knoll JHM, Butler MG Karam S, Lalande M. Genetic imprinting suggested by maternal heterodisomy in nondeletion Prader-Willi syndrome. Nature $1989 ; 342$ : 281-5.

8. Niikawa N, Kajii T. The origin of mosaic Down syndrome: four cases with chromosome markers. Am J Hum Genet 1984 ; 36 : 123-30.

9. Williams J, Wang B, Rubin C, Clark R, Mohandas T. Apparent non-mosaic trisomy 16 in chorionic villi : diagnostic dilemma : clinically significant finding? $A m \mathrm{~J} \mathrm{Hum}$ Genet 1989, 45, suppl. : A 273.

10. Vidaud D, Vidaud M, Plassa F, Gazengel C, Noel B, Goossens M. Father-to-son transmission of hemophilia A due to uniparental disomy. Am J Hum Genet 1989, 45 suppl. A 226.

11. Rockman-Greenberg C, Ray M, Evans JA, Canning M, Hamerton JL. Homozygous Robertsonian translocation in a fetus with 44 chromosomes. Hum Genet 1982; 61 : 181-4.

12. Reik W Genomic imprinting and genetic disorders in man. Trends Genet 1989; 5 : 3316. 